\title{
Identification of 3 Novel Patients with CLCN2-Related Leukoencephalopathy due to CLCN2 Mutations
}

\author{
Burcu Zeydan ${ }^{a, b} \quad$ Ugur Uygunoglu $^{a}$ Ayse Altintas ${ }^{a}$ Sabahattin Saip ${ }^{a} \quad$ Aksel Siva $^{a}$ \\ Truus E.M. Abbink ${ }^{c}$ Marjo S. van der Knaap ${ }^{c}$ Cengiz Yalcinkaya ${ }^{a}$

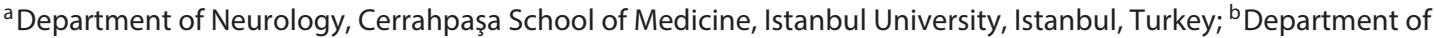 \\ Radiology, Mayo Clinic College of Medicine, Rochester, MN, USA; 'Department of Child Neurology, VU University \\ Medical Centre, Amsterdam, The Netherlands
}

\section{Dear Sir,}

ClC-2 is a chloride channel, which plays an important role in brain ion and water homeostasis both in mice and humans [1]. CLCN2-related leukoencephalopathy $(\mathrm{CC} 2 \mathrm{~L})$ is a recently identified rare disorder, caused by autosomal recessive mutations in CLCN2, which results in $\mathrm{ClC}-2$ chloride channel dysfunction and myelin microvacuolization with characteristic brain MRI features of leukoencephalopathy [2]. We report three patients with CC2L, who carry genetically verified CLCN2 mutations and were presented between 1999 and 2014 to our clinic.

\section{Patients}

All patients were women with adult-disease onset (Table 1). Patient 1 presented with gait-speech difficulty and had dysarthria, nystagmus, ataxic gait and dysmetria. $\mathrm{Pa}$ tient 2 presented with headache, imbalance and blurry vision. She had diplopia, paraparesis and ataxic gait. Patient 3 presented with right-side numbness and ataxic gait.

All laboratory tests were normal. Patient 2 had an abnormal visual evoked po- tential, which showed unilateral P100 latency increase, although the rest of the ophthalmologic examination was normal. All patients had cerebellar ataxia in common and showed distinct MRI findings of bilateral T2-hyperintensities and diffusion restriction in the posterior limbs of the internal capsules, midbrain cerebral peduncles and middle cerebellar peduncles, and low signal in the same localizations on apparent diffusion coefficient (ADC) maps (Fig. 1). After verifying CLCN2 (NM_004366.5) mutations (Table 1), they were diagnosed with CC2L and had stable or very slowly progressive disease courses.

\section{Discussion}

The prevalence of CC2L is not known. Sixteen patients are identified till date, since its discovery in 2013 [2, 3], but there could be more undiagnosed patients existing, who possibly remain asymptomatic. The frequency of the disease should be interpreted cautiously due to the autosomal recessive nature of $\mathrm{CC} 2 \mathrm{~L}$, the relatively high consanguinity in our country and our clinic being a referral center. As an ideal approach, a multicenter MRI database may identify potential individuals with CC2L, who could further be evaluated for genetic mutations.

CC2L is characterized by mild and nonspecific neurological involvement, visual impairment and male infertility $[4,5]$. Mild cerebellar ataxia with action tremor-gait instability is the most common neurological finding, and patients are reported to remain ambulatory during follow-up [2]. The highly variable clinical presentations and age of onset may be partially related to the extent of ClC-2 loss of function [2]. However, the current knowledge on CC2L from the small number of affected individuals does not favor any genotype-phenotype correlations [3]. In line with this, patients 2 and 3 may be expected to have a larger impact on the molecular function of their ClC-2 channels due to the mutations identified, but they had milder clinical phenotypes compared with patient 1 . The correlation between the functional impact of the identified mutations and clinical phenotypes needs to be investigated further as more patients with CC2L are identified.

\section{KARGER}

(c) 2017 S. Karger AG, Basel
Dr. Cengiz Yalcinkaya Department of Neurology Cerrahpasa School of Medicine Istanbul University, Fatih, TR-34098 Istanbul (Turkey) E-Mail cengiz@ istanbul.edu.tr 

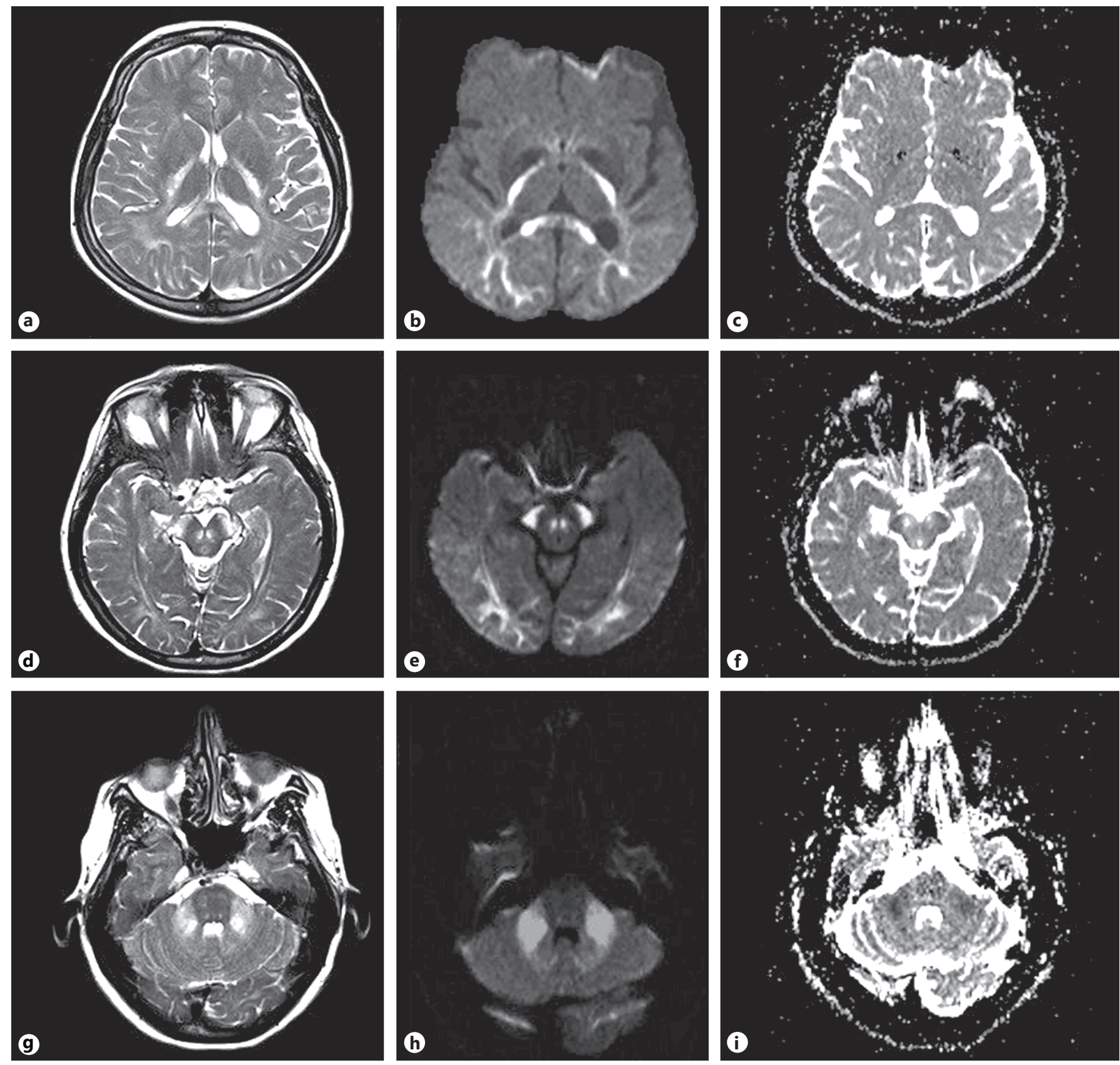

Fig. 1. Brain MRI of patient 1: signal abnormalities in the posterior limbs of the internal capsules (a-c), midbrain cerebral peduncles $(\mathbf{d}-\mathbf{f})$ and middle cerebellar peduncles $(\mathbf{g}-\mathbf{i})$. Hyperintensities

on axial T2-weighted images $(\mathbf{a}, \mathbf{d}, \mathbf{g})$, diffusion restriction on axial diffusion-weighted images $(\mathbf{b}, \mathbf{e}, \mathbf{h})$ and corresponding low signal on axial apparent diffusion coefficient maps $(\mathbf{c}, \mathbf{f}, \mathbf{i})$.

Major inclusion criteria for brain MRI are abnormally high signal on T2-weighted images and abnormally low signal on T1weighted images in the posterior limbs of the internal capsules, midbrain cerebral peduncles and middle cerebellar peduncles [3]. MRI shows diffusion restriction and low ADC values [2] in the same structures as a result of myelin microvacuolization [3]. In adult patients, the diffusion restriction is observed in the specific white matter structures, whereas in pediatric patients there is relatively a more diffuse diffusion restriction [2].
The differential diagnosis for CC2L depends mostly on bilateral abnormal MRI findings, since clinical features are usually nonspecific. The list primarily includes autosomal-dominant adult onset leukodystrophy related to $L M N B 1$ duplications, CMT1X - gap junction beta-1 (GJB1) gene 
Table 1. Demographics and clinical features

\begin{tabular}{|c|c|c|c|}
\hline & Patient 1 & Patient 2 & Patient 3 \\
\hline Age/sex & 47/ Woman & 33/ Woman & 47/ Woman \\
\hline $\begin{array}{l}\text { Early psychomotor development } \\
\text { and cognitive level }\end{array}$ & Normal & Normal & Normal \\
\hline Presenting signs & Gait difficulty, imbalance & $\begin{array}{l}\text { Imbalance, headache, blurry } \\
\text { vision }\end{array}$ & Numbness, imbalance \\
\hline Vision acuity & Normal & Normal & Normal with eyeglasses \\
\hline Visual fields & Normal & Normal & Normal \\
\hline Ataxia & Moderate & Mild & Mild \\
\hline CLCN2 (NM_004366.5) mutations & $\begin{array}{l}\text { Homozygous c. } 1412 \mathrm{G}>\mathrm{A} \text {, } \\
\text { p.Arg471His mutation } \\
\text { MM }\end{array}$ & $\begin{array}{l}\text { Homozygous } \\
\text { p.Glu475LysfsTer79 } \\
\text { FSM }\end{array}$ & $\begin{array}{l}\text { Compound heterozygous } \\
\text { p.Leu435ArgfsTer7 } \\
\text { FSM }\end{array}$ \\
\hline
\end{tabular}

$\mathrm{MM}=$ missense mutation; $\mathrm{FSM}=$ frame shift mutation.

mutation encoding connexin 32 [6], and Pelizaeus-Merzbacher like disease (PMLD) [3].

In conclusion, the MRI findings are essential for the diagnosis of CLCN2-related leukoencephalopathy, a novel and rare disorder caused by CLCN2 mutations with nonspecific clinical findings, distinct MRI patterns and further features yet to be discovered.

\section{References}

1 Blanz J, Schweizer M, Auberson M, et al: Leukoencephalopathy upon disruption of the chloride channel ClC-2. J Neurosci 2007;27: 6581-6589.

2 Depienne C, Bugiani M, Dupuits C, et al: Brain white matter oedema due to $\mathrm{ClC}-2$ chloride channel deficiency: an observational analytical study. Lancet Neurol 2013;12:659_ 668.

\section{Acknowledgments}

The authors would like to thank the Department of Child Neurology, VU University Medical Center, Amsterdam, The Netherlands and Cerrahpaşa Medical Faculty Neurological Research Supporting Association.

3 van der Knaap MS, Depienne C, Sedel F, et al: CLCN2-Related Leukoencephalopathy. GeneReviews ${ }^{\circledR}, 2015$.

4 Bösl MR, Stein V, Hübner C, et al: Male germ cells and photoreceptors, both dependent on close cell-cell interactions, degenerate upon ClC-2 $\mathrm{Cl}(-)$ channel disruption. EMBO J 2001;20:1289-1299.

\section{Statement of Ethics}

Informed consent form was obtained from each individual.

\section{Disclosure Statement}

The authors have no conflicts of interest to declare.
Di Bella D, Pareyson D, Savoiardo M, et al: Subclinical leukodystrophy and infertility in a man with a novel homozygous CLCN2 mutation. Neurology 2014;83:1217-1218.

6 Siskind C, Feely SM, Bernes S, et al: Persistent CNS dysfunction in a boy with CMT1X. J Neurol Sci 2009;279:109-113. 